\title{
Artificial Neural Networks for Diagnosis of Kidney Stones Disease
}

\author{
Koushal Kumar \\ Department of CSE/IT, Lovely Professional University, Jalandhar, Punjab, India \\ kaushal_kumar302@yahoo.com \\ Abhishek \\ Department of CSE/IT, Lovely Professional University, Jalandhar, Punjab, India \\ Bhardwajabhishek786@gmail.com
}

\begin{abstract}
Artificial Neural networks are often used as a powerful discriminating classifier for tasks in medical diagnosis for early detection of diseases. They have several advantages over parametric classifiers such as discriminate analysis. The objective of this paper is to diagnose kidney stone disease by using three different neural network algorithms which have different architecture and characteristics. The aim of this work is to compare the performance of all three neural networks on the basis of its accuracy, time taken to build model, and training data set size. We will use Learning vector quantization (LVQ), two layers feed forward perceptron trained with back propagation training algorithm and Radial basis function (RBF) networks for diagnosis of kidney stone disease. In this work we used Waikato Environment for Knowledge Analysis (WEKA) version 3.7.5 as simulation tool which is an open source tool. The data set we used for diagnosis is real world data with 1000 instances and 8 attributes. In the end part we check the performance comparison of different algorithms to propose the best algorithm for kidney stone diagnosis. So this will helps in early identification of kidney stone in patients and reduces the diagnosis time.
\end{abstract}

Index Terms - Kidney Stone Disease, Multilayer Perceptrons, Radial Basis Function Networks, Learning Vector Quantization, Diagnosis

\section{Introduction}

Kidney stones disease is becoming more and more common now days. Kidney stones are created when certain substances in urine including calcium, oxalate, and sometimes uric acid crystallize. These minerals and salts form crystals, which can then join together and form a kidney stone. Each type of kidney stone has a different cause [1]. Stones are classified according to their chemical composition. Approximately $80 \%$ of all kidneys stones are calcium oxalate stones, which are the most problematic. The formation of these stones may be caused by genetic factors and also depends upon age, and geographical factors [2]. However, more important are dietary and lifestyle factors, and the results of acquired metabolic defects leading to crystal formation and growth of a kidney stone [3]. In [2] authors give a detail explanation regarding what are kidney stones, its Types and different symptoms of this disease. In [3] authors describe the different factors like age, sex, race, body weight, ethnicity which may cause of kidney stones.

The most common problem in the Field of automatic diagnostic is the diagnostics using fast and accurate algorithm which doesn't require long time to run and give accurate and correct results [4]. To reduce the diagnosis time and improve the diagnosis accuracy, it has become more of a demanding issue to develop reliable and powerful medical diagnosis system to support the yet and still increasingly complicated diagnosis decision process. The medical diagnosis by nature is a complex and fuzzy cognitive Process hence soft computing methods, such as neural networks, have shown great potential to be applied in the development of medical diagnosis. In disease diagnosis the learning and detection of partial disease can be helpful when time and information constraints are present. Thus artificial neural networks provide a good means to partial diagnosis.

This we used three neural networks algorithms for measuring their classification accuracy against time taken to classify for diagnosis purpose. This paper is thus organized as following in section II a brief introduction of the artificial neural network, in section III Previous related work works that had been done, in section IV kidney stone data set that is used in this research has been discussed, in section $\mathrm{V}$ artificial neural networks classifiers used is described, In section VI simulation tool used is described, in section VII experiment results and discussion is given and in the last section we conclude the paper. 


\section{Artificial Neural Networks Introduction}

Artificial neural networks (ANN) have emerged as a result of simulation of biological nervous system, such as the brain on a computer. Artificial Neural networks are represented as a set of nodes called neurons and connections between them. The connections have weights associated with them, representing the "strength" of those connections. Nowadays neural networks can be applied to problems that do not have algorithmic solutions or problems for which algorithmic solutions are too complex to be found. In others words the kind of problems in which inputs and outputs variables does not have a clear relationship between them, a neural networks is a efficient approach in such problems. Most neural network architecture has three layers in its structure. First layer is input layer which provides an interface with the environment, second layer is hidden layer where computation is done and last layer is output layer where output is stored. Data is propagated through successive layers, with the final result available at the "output layer". Many different types of neural networks are available and multi layer neural networks are the most popular. MLP popularity is due to more then one hidden layer in its structure which helps sometimes in solving complex problems which a single hidden layer neural network cannot solve.

Figure 1 show multilayer perceptron structure with $\mathrm{N}$ number of inputs neurons corresponding to $\mathrm{N}$ number of hidden and output neurons.

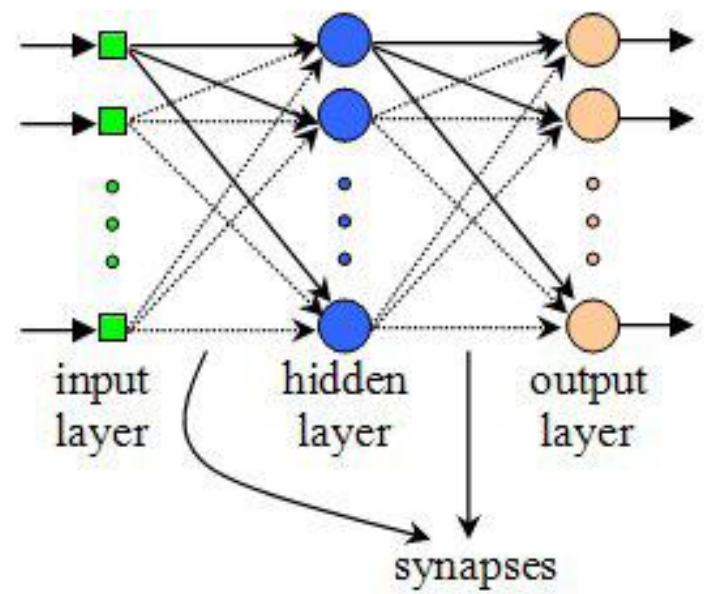

Figure 1: Multilayer Perceptron architecture

Nowadays artificial neural network has become most widely tool used for diagnosis of diseases. Because of the Fault tolerance, Generalization and Learning from environment like capabilities of Artificial neural networks it is becoming more and more popular in medical diagnosis and many more others areas. One of the network structures that have been widely used is the feed forward network where network connections are allowed only between the nodes in one layer and those in the next layer. Feed-forward back propagation neural network is used as a classifier to distinguish between infected or non-infected person.
Figure 2 shows the architecture of feed forward neural networks (MLP) for decision making. In this structure three inputs are provided to network and then inputs and weights are summed by using summation function. Finally output is in binary form either Yes or No. Yes for a patient who is suffering from disease and no for unaffected person. In this work three neural network algorithms LVQ, RBF and feed forward architecture with back propagation algorithms have been investigated for diagnosis of kidney stones for early detection of disease. All three algorithms are compared against their classification accuracy to classify affected and unaffected persons.

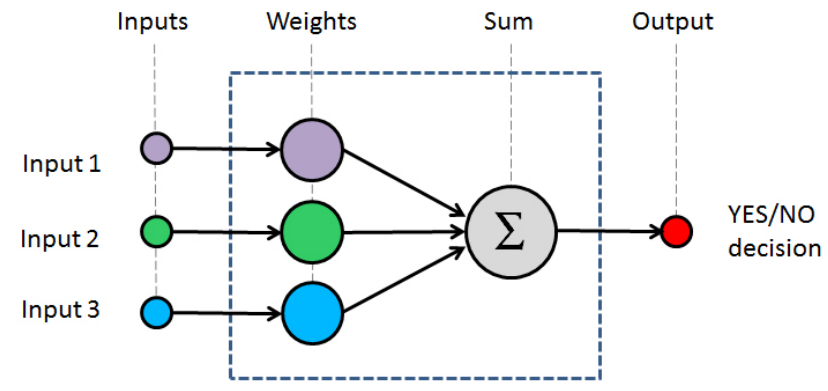

Figure 2 Feed Forward Architecture in Decision Making

\section{Related Work}

There is a continuous study and research going on in this field of medical diagnosis. There is lot of work has been done on diseases like Cancer, Diabetes, Heart attack etc using neural networks. Koizumi.N et al (2011) presents a" Robust kidney stone tracking for a noninvasive ultrasound theragnostic system" propose a noninvasive ultrasound theragnostic system that tracks movement in an affected area (kidney stones, in the present study) by irradiating the area with highintensity focused ultrasound [5]. Mitri F.G. (2011) presents "Vibro-acoustography imaging of kidney stones in vitro Vibro-acoustography" (VA) is an ultrasound-based modality sensitive to stiffness and free from speckle and possesses some advantages over conventional ultrasound imaging in terms of image quality [6]. Duryeal A.P. et al. (2010) presents an "Optimization of Histotripsy for Kidney Stone Erosion Histotripsy" is a technique for the mechanical fractionation of tissue structures which utilizes focused pulsed-ultrasound to direct the activity of a cavitational bubble cloud [7]. Rouhani $M$ et al. (2009) present the "Comparison of several Ann architecture RBF, GRNN, PNN, LVQ and SVM on Thyroid Disease". The performance of each architecture is studied, and the best method is selected for each of classification tasks. In this paper RBF and PNN selected best models for diagnosis [8]. Shukla A. et al (2009) presents" Knowledge Based Approach for Diagnosis of Breast cancer" this paper presents a novel approach to simulate a Knowledge Based System for diagnosis of Breast cancer using Ann and apply three neural networks algorithms BPA, RBF and LVQ on the disease 
and find best model for diagnosis. In this paper LVQ select as a best model for diagnosis on the disease and find best model for diagnosis [9].

\section{Kidney Stone Dataset}

The dataset of diagnosis for kidney stone disease is purely real set data. The data set used in this work is collected from different medical laboratories which test the patients of kidney stones. In this work the 1000 patients data have been used i.e. 1000 instances and has 7 attributes. The attributes are actually symptoms of kidney stone on the basis of which we trained neural networks for diagnosis. The attributes taken for diagnosis are Lymphoctyes, Monocytes, Eosinophis, Neutrophil, S. Creatinine, Blood Sugar, and U.Acid. The dataset are divided into two classes to facilitate their use in experimentally determining the presence or absence of kidney stone disease. The class 1 is containing yes value mean presence of disease and class 2 containing No value means absence of disease

Table 1 Sample Report for Kidney Stone

\begin{tabular}{|c|c|c|}
\hline ATTRIBUTES & WEIGHT & ACTUAL RANGE \\
\hline LYMPHOCTYES & $30 \mathrm{gms}$ & $20-50 \%$ \\
\hline MONOCYTES & $01 \mathrm{gms}$ & $1-6 \%$ \\
\hline NEUTROPHIL & $02 \mathrm{gms}$ & $1-4 \%$ \\
\hline S. CREATININE & $61 \mathrm{gms}$ & $50-70 \%$ \\
\hline EOSINOPHIS & $3 \mathrm{gms}$ & $4-10 \%$ \\
\hline
\end{tabular}

The attributes present in Table 1 shows that if these parameters with in actual range of attribute its means the person is suffering from kidney stone i.e. containing class YES otherwise containing NO class.

\section{Artificial Neural Networks Classifiers Used}

The main aim of this work is to compare the classification performance of three different neural networks algorithms in diagnose of kidney stones disease. The algorithms used are Radial basis functions (RBF), Learning Vector Quantization (LVQ) and a Multilayer perceptron with back propagation algorithm. A brief description of them is as follow.

\section{A) Radial Basis Function Networks}

A Radial basis function (RBF) network is a special type of neural network that uses a radial basis function as its activation function. A Radial Basis Function (RBF) neural network has an input layer, a hidden layer and an output layer. The neurons in the hidden layer contain radial basis transfer functions whose outputs are inversely proportional to the distance from the center of the neuron [10]. RBF networks are very popular for function approximation, curve fitting, Time Series prediction, and control problems. Because of more compact topology then other neural networks and faster learning speed, RBF networks have attracted considerable attention and they have been widely applied in many science and engineering fields [11-14]. A general block diagram of an RBF network is illustrated in Figure 3. In RBF networks, the outputs of the input layer are determined by calculating the distance between the network inputs and hidden layer centers. The second layer is the linear hidden layer and outputs of this layer are weighted forms of the input layer outputs. Each neuron of the hidden layer has a parameter vector called center.

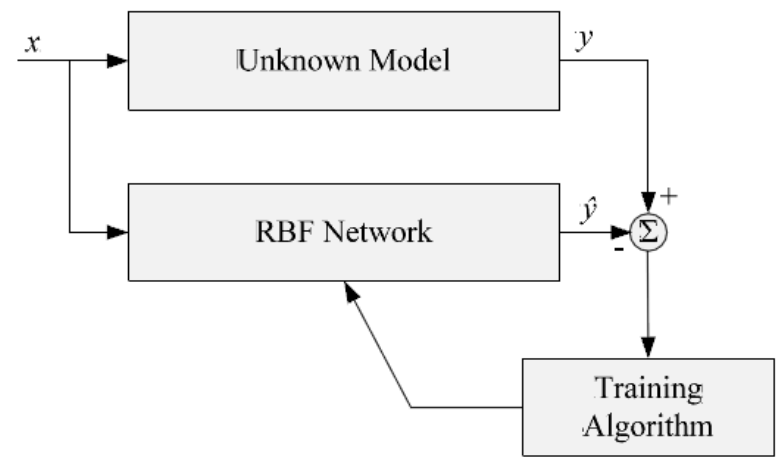

Figure 3: Block diagram of RBF networks [11]

\section{B) Multilayer Perceptron with BPA}

A multilayer perceptron is a feed forward artificial neural network model that maps sets of input data onto a set of appropriate output. It is a modification of the standard linear perceptron in that it uses three or more layers of neurons (nodes) with nonlinear activation functions, and is more powerful than the perceptron in that it can distinguish data that is not linearly separable, or separable by a hyper-plane [15]. Backpropogation is the most widely applied learning algorithm for multilayer perceptron in neural networks. Backpropogation employs gradient descent to minimize the squared error between the network output values and desired values for those outputs. These error signals are used to calculate the weight updates which represent knowledge learnt in the networks. The performance of Backpropogation algorithm can be improved by adding a momentum term [16].

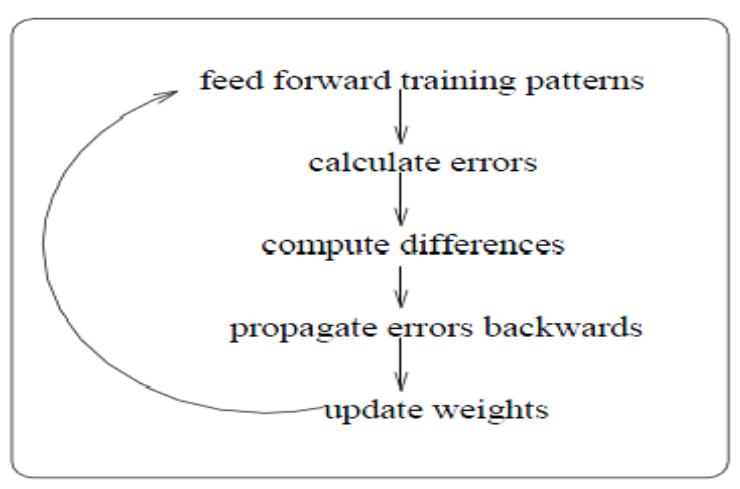

Figure 4. The back-propagation NN epoch cycle[17] 
Figure 4 Show the whole cycle of epoch. In neural networks training the term epoch is used to describe a complete pass through all of the training patterns. The weight in the neural net may be updated after each pattern is presented to the net, or they may be updated just once at the end of the epoch.

\section{C) Learning Vector Quantization Algorithm}

A Learning Vector Quantization Network (LVQ) has a first competitive layer and a second linear layer. The competitive layer learns to classify input vectors in much the same way as the competitive layers of SelfOrganizing Nets. The linear layer transforms the competitive layer's classes into target classifications defined by the user. The classes learned by the competitive layer are referred to as subclasses and the classes of the linear layer as target classes. Both the competitive and linear layers have one neuron per class. LVQ learning in the competitive layer is based on a set of input/target pairs. The second layer needs no learning as the output classes are known for each input pattern [18].

\section{Simulation Tool Used}

The tool used in this work is WEKA version 3.7.5. WEKA is developed by the University of Waikato in New Zealand. WEKA is based upon java platform. WEKA is free software available under the GNU General Public License. In WEKA the algorithms directly apply to the dataset. WEKA implements algorithms for data preprocessing, classification, regression, and clustering and association rules. The result also shows the other functions that obtained after training and testing that are kappa statistics, mean absolute error and root mean squared error in the form of numeric value and relative absolute error and root relative squared error in the form of percentage. The $70 \%$ data is used for training of networks, $15 \%$ data is used for testing of networks and $15 \%$ data is used for validation [19].

\section{Experiment Results and Discussion}

Medical diagnosis by neural network is the blackbox approach. A network is chosen and trained with examples of all classes. After successful training, the system is able to diagnose the unknown cases and to make predictions. In this experiment work we applied three neural networks on kidney stones disease for classification and diagnosis of patients. The results of experiments are shown below in tables. Table II shows neural networks are trained with 1000 instances or data items and testing of neural networks is done with 150 data instances. The numbers of hidden layers used are 2 which are sufficient for any kind of classifications. Learning rate and momentum used for training and testing is 0.3 and 0.2 respectively, this particular value is chosen because of maximum accuracy is achieved with these parameters. Validation threshold has value 20 and value of error per epoch is 0.013 .

Table II: MLP parameters with Maximum Accuracy

\begin{tabular}{|l|c|}
\hline \multicolumn{1}{|c|}{ Parameters } & Values \\
\hline Number of Training Data & 1000 \\
\hline Number of Testing Data & 150 \\
\hline Number of Hidden Layers & 2 \\
\hline Learning Rate & 0.3 \\
\hline Momentum & 0.2 \\
\hline Validation Threshold & 20 \\
\hline Error Per Epoch & 0.013 \\
\hline Accuracy & $98 \%$ \\
\hline
\end{tabular}

Table II shows the parameters we used for training and testing of different networks. Total numbers of instances are 1000 instances used for testing are 150, number of hidden layers are two, learning rate of network is 0.3 and momentum is 0.2 . Learning rate is defined as training parameter that controls the size of weight and bias changes during learning and momentum simply adds a fraction $m$ of the previous weight update to the current one. The momentum parameter is used to prevent the system from converging to a local minimum or saddle point. A high momentum parameter can also help to increase the speed of convergence of the system. However, setting the momentum parameter too high can create a risk of over fitting the minimum, which can cause the system to become unstable.

Table III. Comparison Results of applied algorithms

\begin{tabular}{|c|c|c|c|c|}
\hline ANN's & $\begin{array}{c}\text { Correctly classified } \\
\text { instances }\end{array}$ & $\begin{array}{c}\text { Incorrectly classified } \\
\text { instances }\end{array}$ & Time taken in sec & Accuracy \\
\hline MLP & 977 & 23 & 0.44 & $92 \%$ \\
\hline RBF & 925 & 75 & 0.42 & $87 \%$ \\
\hline LVQ & 910 & 90 & 0.57 & $84 \%$ \\
\hline
\end{tabular}


Table III shows that multilayer perceptron trained with back propagation algorithm classified most of instances correctly and give accuracy of $92 \%$, and time taken is $0.44 \mathrm{sec}$. The numbers of hidden layers for multilayer perceptron used is two, learning rate and momentum of MLP used is 0.3 and 0.2 respectively. RBF classified $87 \%$ of instances correctly and take less time for classification then MLP, but error rate is more in RBF as compare to MLP. LVQ classified $84 \%$ of instances correctly and take more time then other networks. Many researchers reported that the performance of Radial basis function networks is better than that of multilayer perceptrons for their applications, but some other researchers reported the opposite results. But in our work the performance of RBF is good only in the condition of small data set as we keep on increasing the instances of data set the performance of RBF deteriorate.

Table IV: Results of classification after Training and Testing

\begin{tabular}{|c|c|c|c|c|c|}
\hline ANN's & Kappa statistics & Mean Absolute Eroor & Root Mean Square & Relative Absolute Error & Root Relative Eroor \\
\hline MLP & 0.9613 & 0.0283 & 0.1207 & $5.710 \%$ & $24.26 \%$ \\
\hline RBF & 0.8732 & 0.0774 & 0.205 & $15.63 \%$ & $41.21 \%$ \\
\hline LVQ & 0.8459 & 0.0751 & 0.274 & $15.17 \%$ & $55.08 \%$ \\
\hline
\end{tabular}

Table IV shows different parameters which play critical role in the accuracy and performance of the neural networks. Kappa statistics is a chance-corrected measure of agreement between the classifications and the true classes. It's calculated by taking the agreement expected by chance away from the observed agreement and dividing by the maximum possible agreement. Rests of all parameters are kind of errors [20].

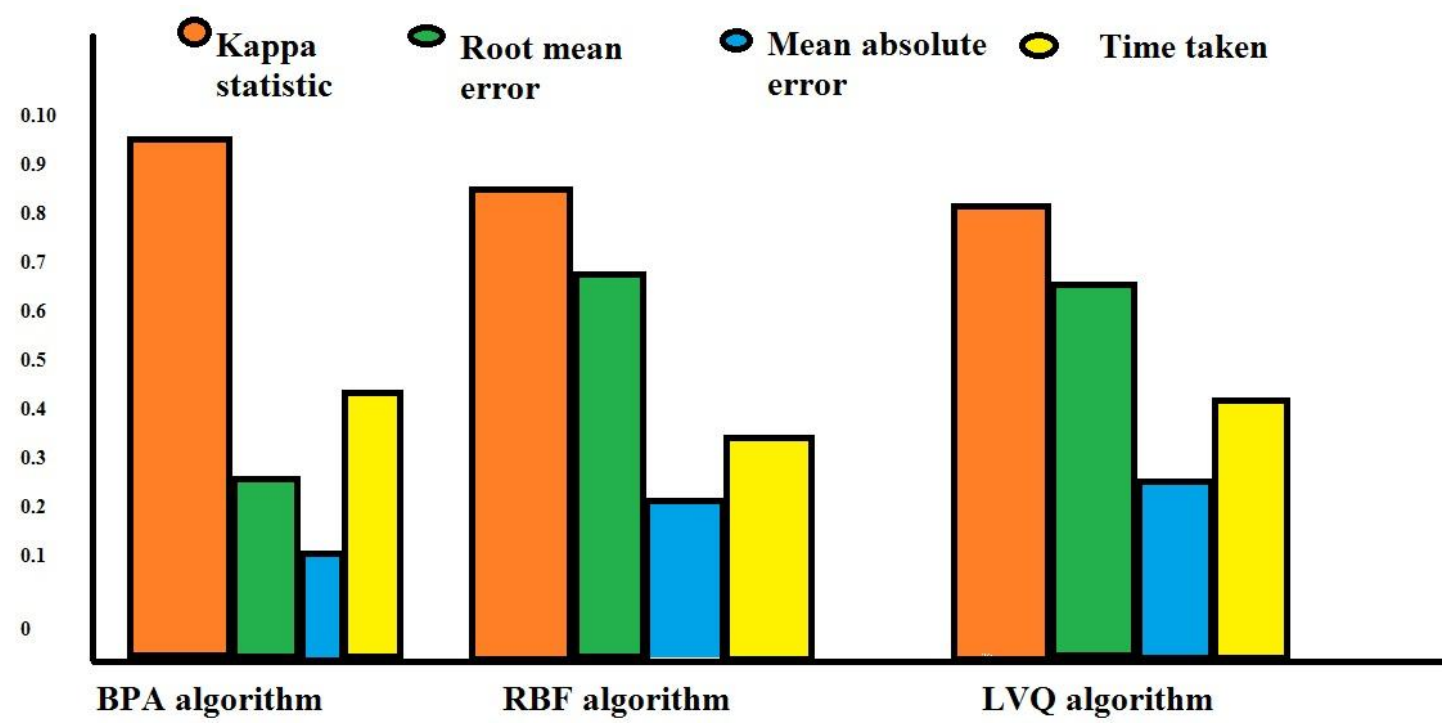

Figure 5: Performance comparison of algorithms

\section{Conclusion}

In the Work Presented Here, Three Neural Network Algorithms have been investigated for Diagnosis Of kidney stones diseases. From the above results we achieve our objective to find the best model for kidney stone diagnosis. The multilayer perceptron with two hidden layers and back propagation algorithm is the best model for diagnosis of kidney stone disease. Its accuracy is $92 \%$ to diagnosis the kidney stone disease. It correctly classified the 977 instances from 1000 instances. Neither of other networks under consideration are superior in terms of classification accuracy to multilayer perceptron trained with back propagation algorithm. Even the error in MLP is less then other two algorithms. Thus finally we come to conclusion that multilayer perceptron trained with back propagation is best algorithm for kidney stones diagnosis.

\section{References}

[1] http://www.webmd.com/kidneystones/understandi ng- kidney-stones-basics

[2] Moe OW. Kidney stones: pathophysiology \& Medical management. Lancet 2006; 367: 333-44.

[3] Sandhya A et al "Kidney Stone Disease Etiology And Evaluation" Institute of Genetics and Hospital for Genetic Diseases, India International Journal of Applied Biology and Pharmaceutical Technology,may june 2010 
[4] http://www.internage.kicv.ua/projects/neuraln/.htm 1

[5] Koizumi N et al "Robust Kidney Stone Tracking for a Non-invasive Ultrasound" Shanghai International Conference Center May 9-13, 2011, Shanghai, China

[6] Mitri F.G. "Vibro-acoustography imaging of kidney stones in vitro" IEEE Transactions on Biomedical Engineering 2011

[7] Duryeal A.P. et al. "Optimization of Histotripsy for Kidney Stone EROSION Department of Biomedical Engineering, University of Michigan, Ann Arbor, MI 2Department of Urology, University of Michigan, Ann Arbor, MI 2010

[8] Rouhani M. et al. "The comparison of several ANN Architecture on thyroid disease"IslamiAzadUniversity, Gonabad branch Gonabad 2010

[9] Shukla A. et al "Diagnosis of Thyroid Disorders using Artificial Neural Networks" Department of Information Communication and Technology, ABV-Indian Institute of Information Technology and Management Gwalior, India IEEE International Advance Computing Conference 2009

[10] Broomhead, D; Low, D. Multivariable functional interpolation and adaptive networks. Complex Systems 1988, 2, 321-355.

[11] Tuba Kurban and Erkan Beşdok. "A Comparison of RBF Neural Network Training Algorithms for Inertial Sensor Based Terrain Classification" Geomatics Engineering, Engineering Faculty, Erciyes University, Turkey 2009

[12] Devaraj, D.; Yegnanarayana, B.; Ramar, K. Radial basis function networks for fast contingency ranking. Electric. Power Energy Syst. 2002, 24, 387-395.

[13] Fu, X. Wang, L. Data dimensionality reduction with application to simplifying rbf network structure and improving classification performance. IEEE Trans. Syst. Man Cybern. Part B 2003,33, 399-409.

[14] Han, M.; Xi, J. Efficient clustering of radial basis perceptron neural network for pattern recognition. Pattern Recognit 2004, 37, 2059-2067.

[15] Rohitash chandra, Kaylash Chaudhary and akshay kumar.,2007 The combination and comparison of neural networks with decision trees for wine classification. school of sciences and technology.

[16] Jamal M. Nazzal, Ibrahim M. El-Emary and Salam A. Najim." Multilayer Perceptron Neural Network (MLPs) For Analyzing the Properties of Jordan Oil Shale. World Applied Sciences Journal 5 (5): 546552,2008
[17] Koushal Kumar, Gour Sundar Mitra Thakur. "Extracting Explanation from Artificial Neural Networks" International Journal of Computer Science and Information Technologies, Vol.3 (2) 2012, 3812- 3815.

[18] Modjtaba Rouhani and Mehdi motavalli haghighi. "The Diagnosis of Hepatitis diseases by Support Vector Machines and Artificial Neural Networks.International Association of Computer Science and Information Technology - Spring Conference 2009

[19] WEKA at http://www.cs.waikato.ac.nz/ ml/weka.

[20] http://www.dmi.columbia.edu/homepages/chuangj /kappa

\section{Short Bio Data for the Author's}

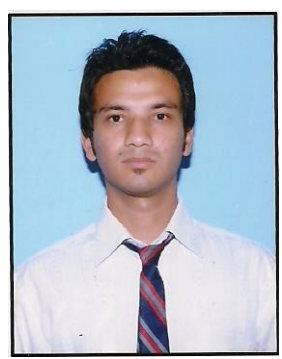

Mr. Koushal Kumar Has done his M.Tech degree in Computer Science and Engineering from Lovely Professional University, Jalandhar, India. He obtained his B.S.C and M.S.C in computer science from D.A.V College Amritsar Punjab. His area of research interests lies in Artificial Neural Networks, Soft computing, Computer Networks, Grid Computing, and data base management systems

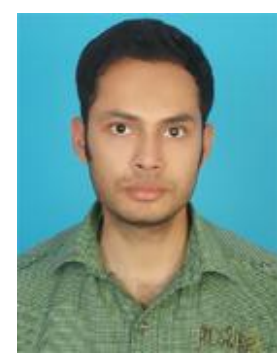

Mr. Abhishek Has done his M.Tech degree in computer science and Engineering from Lovely Professional University, Jalandhar, India. He obtained his B.Tech (IT) degree from DAV collage of Engg \& Technology Jalandhar. His Area of interest are Artificial Neural Network, Computer networking and Data structure. 